Faculty of Mathematical Sciences

\section{University of Twente}

University for Technical and Social Sciences
P.O. Box 217

7500 AE Enschede

The Netherlands

Phone: +31-53-4893400

Fax: +31-53-4893114

Email: memo@math.utwente.nl

Memorandum No. 1474

Mass transport in a partially

covered fluid-filled cavity

C.H. Driesen, J.G.M. Kuerten and H.K. Kuiken

DeCember 1998

ISSN 0169-2690 


\title{
MASS TRANSPORT IN A PARTIALLY COVERED FLUID-FILLED CAVITY
}

\author{
C.H. DRIESEN, J.G.M. KUERTEN AND H.K. KUIKEN \\ Twente Institute of Mechanics, J.M. Burgers Centre \\ Faculty of Mathematical Sciences \\ University of Twente, P.O. Box 217 \\ 7500 AE Enschede, The Netherlands
}

\begin{abstract}
A method of computing the concentration field of dissolved material inside an etch-hole is presented. Using a number of assumptions, approximate convectiondiffusion equations are formulated, and analytical descriptions for the concentration in different parts of the domain are obtained. By coupling these descriptions the concentration field can be computed. The assumptions and the results are validated by comparison with solutions based on a finite-volume method. Results of the boundary-layer method are given for two characteristic etch-hole geometries. The described boundarylayer method is efficient in terms of computational time and memory, because it does not require the construction of a computational grid in the interior of the domain. This advantage will be exploited in a future paper where the method will be used to simulate wet-chemical etching.
\end{abstract}

Key words: boundary layers, special functions, etching, cavity, mass transfer Mathematics Subject Classification: 33C10, 45F05, 76D10, 80A20

\section{INTRODUCTION}

The mathematical modelling of fluid flow in combination with mass or heat transport in cavities has been a topic of active research for the last three decades. Several articles have been written for a wide range of applications varying from the evolution of corrosion pits, heat transfer along rough surfaces, crystal-growth processes, electro-deposition, and mass transfer from cavities in artery walls.

In this paper, a mathematical model and a numerical method are developed for modeling mass transport in a partially covered cavity as a part of a numerical simulation method for wet-chemical etching. The material and fluid-flow properties of this process are used to simplify the convection-diffusion equation. In this way a time- and memory-saving algorithm for the computation of the concentration of dissolved material in arbitrary etch-hole geometries can be 
obtained. Although the description of the method is given in this context, the mass-transfer results can be translated directly into a heat-transfer analogue.

Wet-chemical etching is an important technique in modern technology. Applications can be found in the production of oil filters, shadow masks for color TV sets, lasers, integrated circuits, etc. As another application of wet-chemical etching, we can think of the etching procedure of small grooves in rotating disks, such as depicted in Fig. 1. These disks are used as electrodes. When the width $W$ of the groove is small in comparison to the distance to the center of the disk, a two-dimensional approximation can be made. A typical etching process can be described in the following way (see Fig. 2): A thin piece of metal is partly covered with a mask. A chemical solution is sprayed onto it, dissolving the exposed metal. Using this method, detailed small products can be produced routinely. Because dissolution will occur in all directions, geometries such as that depicted in Fig. 3 will arise. Sideways etching under the masks results in the socalled 'undercut' effect. The result of this effect is that the etch hole is larger, and the size of the etched product is smaller than the original mask pattern. These differences between products and masks can become large, especially for products whose sizes are of the same order as the thickness of the material. Therefore, research is necessary to obtain information about this effect in etching processes.

The modelling of heat or mass transport from cavities and the related simulation of wetchemical etching have been considered in a number of studies. In $[15,16,17,28]$ the etching process was modelled by a diffusion equation. For such a process, in which the effect of fluid flow is disregarded, some analytical solutions for the propagating etch-hole boundary $[15,16,17]$ were found. In $[16,28]$ numerical methods were introduced to tackle this problem. However, in most of the industrial wet-chemical etching applications, convection plays an important role. One can think of a set-up in which the etchant flows along the mask surface, thus creating a flow pattern inside the etch hole. This flow pattern may have a large influence on the time evolution of the etch-hole boundary. Therefore, it is important to obtain accurate numerical simulations of convection-driven etching processes.

The diffusion coefficient of a metal in a wet-chemical etching process is typically of the order of $10^{-10} \mathrm{~m}^{2} \mathrm{~s}^{-1}$. Therefore the etching simulation can be performed in a quasi-stationary manner. Since the boundary of the etch hole moves slowly in comparison with the fluid, the velocity and concentration fields at each time step can be computed as if the boundary were stationary. If the fluid has a uniform density in the etch-hole, the flow problem can be solved independently of the concentration problem. Several papers were written based on finite-element or finite-volume approaches $[2,9,11,12,13,24,25,26]$ for the computation of the fluid flow in an etched cavity. In [23] a boundary-element method is used. Afterwards the concentration field is computed with a finite-volume, finite-element or spectral method. If the concentration gradient normal to the wall is known, the displacement of the etch-hole boundary can be computed.

In the cited numerical simulations, as well as in experiments $[2,7,8,11,13]$, it is noticed that the etch speed decreases when the depth-width ratio of the cavity increases. When the etching has gone down to a depth that is comparable to the width of the orifice of the cavity, an eddy will emerge inside the etch hole. The Péclet number, which is the ratio of the characteristic 
length scale multiplied by the velocity and divided by the diffusion coefficient

$$
P e=\frac{U L}{D},
$$

is an important parameter for the qualitative description of mass transport. For large values of the Péclet number transport of the 'polluted' fluid takes place in a thin boundary layer along the rim of this eddy $[9,11,14,23,26]$. The exchange of etching products occurs by means of diffusion across the streamline that separates the eddy from the outer flow. Around the center of the eddy the concentration of the dissolved material is uniform. Therefore, only the concentration in the rims of the eddies needs to be computed.

In this paper, we describe a time- and memory-saving method by which the concentration field can be computed around these rims. We extend the analytical descriptions of the concentration around eddy rims as introduced by Kuiken in [14]. In the geometry of [14] only one eddy was present. Kuiken derived equations in which the influence of the fluid flow was reduced to one unknown parameter. In the present paper, the theory as described in [14] is validated, an extension to the theory is made, and the method is applied to realistic etch-hole geometries.

Although the method as described in [14] is applicable to a wide range of problems, finitevolume or finite-element approaches were preferred later in papers on the modelling of heat or mass-transfer problems in cavities. In a rare counter-example presented in [4] the authors used the boundary-layer behavior of heat and mass transfer at high Péclet numbers to obtain an integral description in a radial creeping flow.

When a numerical simulation of wet-chemical etching is constructed in a quasi-stationary way by means of a finite-volume or finite-element method, a large number of equations must be solved at each time step. In this way, a time-consuming etch simulation is obtained. Especially, when the Péclet number increases, the convergence of such methods decreases, and this makes it even more difficult to compute a solution to the concentration problem.

The method presented here has the advantage that it does not need much computational time or memory. The only information needed is the normal stress at the etch-hole boundary and the location of, and the velocity components at, the dividing streamlines. A computational grid in the etch-hole geometry is not necessary. Another advantage of this method is that we can use a boundary-element method to obtain all information needed for the computation of the concentration. Normally a boundary-element method is expensive for such a problem, because the velocity components have to be computed in many interior points in order to solve the convection-diffusion equation with a finite-volume method. In the method described here, the velocity components are only needed at a few interior points. In this way, a numerical simulation of etching can be constructed which needs little computational time and memory in comparison with earlier numerical schemes.

\section{Mathematical Formulation}

In this section, we formulate the mathematical method used in this paper. In the first subsection we give the definition of the problem. In the second subsection, a boundary-layer analysis of the concentration of the dissolved material is used to simplify the convection-diffusion equation. 


\subsection{Problem definition}

In this paper we consider the equations describing the concentration field of a dissolved material in a given velocity field. We assume that the diffusion coefficient $D$ is constant. With this assumption, the concentration field $c$ is described by the stationary convection-diffusion equation:

$$
\boldsymbol{u} \cdot \nabla c-D \Delta c=0
$$

where $\boldsymbol{u}$ is the velocity vector. In this paper we describe a method by which the concentration of dissolved material can be computed for an arbitrary etch-hole geometry. An example of such an etch-hole geometry is given in Fig. 3. The concentration has a constant value $c_{w}$ at the etch-hole boundary. The dissolved material cannot penetrate the mask, and the normal gradient is taken equal to zero, i.e.

$$
\frac{\partial c}{\partial n}=0
$$

Above the etch-hole, the boundary conditions depend on the direction of the fluid flow. In the oncoming fluid, the concentration of the dissolved material is equal to $c_{0}$. In the fluid flowing out of the cavity, convection is dominant. Therefore, boundary conditions are not necessary in the outflow region.

\subsection{Assumptions and simplifications}

In this subsection we describe the assumptions that may be used to simplify the convectiondiffusion equation for different sub-domains. The simplified equations can be solved in a semianalytical way.

In the case of a uniform shear flow along an etch hole, eddies will arise in the interior. The method we use is applicable for an arbitrary number of eddies. In fact, it is known [21] that there exists an infinite sequence of eddies of diminishing strength near sharp corners. It was concluded in [14] that the influence of these very small eddies would but slightly improve the results. As an example, in Fig. 3 only three eddies are shown, the smaller vortices in the sharp corners are disregarded.

Due to the convection-dominated character of the process, the concentration will only vary in thin boundary layers along the rims of the eddies, which are drawn with the dashed lines in Fig. 4. The method starts with the splitting of these eddy rims into different sub-domains. When we consider, for example, the central eddy in Fig. 4, the fluid near the outside of this eddy flows first along the etch-hole boundary (boundary layer 2) and then along the free stream line (number 7 in Fig. 4). The eddy boundary layer is split at such a change in boundary condition. In this way, three different kinds of boundary layers are obtained: There are free boundary layers, boundary layers along the masks, and boundary layers along the etch-hole boundary. We will illustrate the simplification of the convection-diffusion equation for these three different kinds.

For all boundary layers, we assume that the diffusion in streamwise direction is negligible when compared to the diffusion in normal direction. We neglect the curvature for the local 
coordinate systems, since the radius of curvature is asymptotically large in comparison with the thickness of the boundary layers.

As a first example, we consider a free boundary layer, numbered 7 in Fig. 4 . The local system $(x, y)$ is introduced as shown in Fig. 5. Here $y$ is the distance normal to the dividing streamline and $x$ measures distance along the streamline. Variations of the velocity component tangential to the separating streamline in the direction normal to this streamline are ignored. Using the continuity equation, we may derive an expression for the normal velocity component. Here we should remark that the thickness of a boundary layer depends on the Péclet number. A high Péclet number will result in a thin boundary layer. The assumptions regarding the velocity profiles are realistic for large Péclet numbers. When all assumptions are used to simplify (2.2), we obtain $[14]$

$$
u_{7}(x) \frac{\partial c_{7}}{\partial x}-y u_{7}^{\prime}(x) \frac{\partial c_{7}}{\partial y}=\kappa \frac{\partial^{2} c_{7}}{\partial y^{2}},
$$

where $u_{7}(x)$ is the tangential velocity component along $y=0, \kappa$ is the diffusion coefficient scaled by the lengthscale of the cavity and the fluid velocity, and 7 is the index of the boundary layer. The region where this equation holds is formally defined by $0 \leq x \leq x_{7}$ and $-\frac{1}{2} \delta_{y} \leq y \leq \frac{1}{2} \delta_{y}$, where $x_{7}$ is the length and $\delta_{y}$ is the thickness of the boundary layer. The boundary conditions for the concentration in this free boundary layer are

$$
\frac{\partial c_{7}}{\partial y}=0 \quad \text { at } \quad y=-\frac{1}{2} \delta_{y} \text { and } y=\frac{1}{2} \delta_{y}, \quad 0 \leq x \leq x_{7} .
$$

The free-surface conditions at $x=0$ are derived from the boundary-layer joints, and will be given later.

As a second example we consider a boundary layer along the mask, numbered 9 in Fig. 4 . In Fig. 5, the local coordinates $(q, n)$ are shown. Here $n$ is the distance normal to the mask and $q$ measures distance along the mask. For these boundary layers, we assume that the tangential velocity component has a linear profile near the boundaries. Substitution of the assumptions in (2.2) results in [14]

$$
\alpha_{9}(q) n \frac{\partial c_{9}}{\partial q}-\frac{1}{2} \alpha_{9}^{\prime}(q) n^{2} \frac{\partial c_{9}}{\partial n}=\kappa \frac{\partial^{2} c_{9}}{\partial n^{2}}
$$

where $\alpha_{9}(q)$ is the shear stress at the mask. The region where this description holds is $0 \leq q \leq q_{9}$ and $0 \leq n \leq \delta_{n}$, where $q_{9}$ and $\delta_{n}$ have the same meaning as $x_{1}$ and $\delta_{y}$ for the free boundary layers. The boundary conditions for $c_{9}$ are

$$
\frac{\partial c_{9}}{\partial n}=0 \quad \text { at } n=0 \text { and } n=\delta_{n}, \quad 0 \leq q \leq q_{9} .
$$

The free-surface conditions at $q=0$ will be given near the end of this section.

As a third example we consider a boundary layer along the wall, numbered 2 in Fig. 4 . The assumptions and simplifications are similar to those used for the mask boundary layers. The simplified convection-diffusion equation with boundary conditions can be written as (2.6) in 
combination with (2.7) after substitution of the local coordinates $(\phi, \theta)$. The boundary condition at $\theta=0$ changes into

$$
c_{2}=c_{w} \text { at } \theta=0, \quad 0 \leq \phi \leq \phi_{2},
$$

with $\phi_{2}$ the length of the boundary layer. The only remaining boundary conditions are the joints between the different boundary layers.

For each boundary layer, free-surface conditions are necessary for the concentration profile at the beginning of the boundary layer. From Fig. 4 we see that the concentration at the end of a boundary layer can be related to the concentration at the beginning of the next boundary layer. We assume that the fluid flow is smooth at each layer interface, and the concentration remains the same along the streamlines in those regions. Therefore, a reasonable matching procedure is to relate the concentration profile at the end of one boundary layer to the profile at the beginning of the next boundary layer by means of the stream function $\Psi$, defined as

$$
u=\frac{\partial \Psi}{\partial y}, v=-\frac{\partial \Psi}{\partial x}
$$

where $u$ and $v$ are the velocities in streamwise and transverse direction respectively and $x$ and $y$ are the local coordinates. To show an example, for the region where boundary layer 7 joins boundary layer 2 , we find

$$
c_{7}(x, \Psi) \sim c_{2}(\phi, \Psi) \text { if } \frac{x}{x_{7}} \ll 1 \text { and } \frac{\phi_{2}-\phi}{\phi_{2}} \ll 1 \text {, }
$$

from which the relation

$$
u_{7}(x) y=\frac{1}{2} \alpha_{2}(\phi) \theta^{2}
$$

can be derived. The coupling of the stream-function values is shown in Fig. 6. This way of coupling the profiles will result in an error because the expressions for the stream functions do not hold for $x=0$ and $\phi=\phi_{2}$ respectively. At the region where boundary layer 6 is coupled to boundary layer 5 , we find

$$
\frac{1}{2} \alpha_{6}(\phi) \theta^{2}=\frac{1}{2} \alpha_{5}(q) n^{2} \text { if } \frac{\phi_{1}-\phi}{\phi_{1}} \ll 1 \text { and } \frac{q}{q_{5}} \ll 1 \text {. }
$$

For the other boundary-layer connections, similar relations can be derived except for the concentration profile at the beginning of boundary layer number 1. For this concentration profile, the value of the concentration at $x=0$ and $y \leq 0$ is taken equal to $c_{0}$. Note that boundary layer 3 as well as boundary layer 7 are coupled to four other boundary layers. In total, 12 relations can be derived such as given in (2.10) and (2.11). 


\section{INTEGRAL REPRESENTATIONS}

In this section, we will derive integral representations for the solutions of equations (2.6) and (2.4) in combination with the boundary conditions. This derivation is done for the examples given in the previous section. To obtain integral representations, we introduce the following transformations. For the concentration profiles along free streamline 7, we introduce [14]

$$
\begin{gathered}
c_{7}=c_{0}+\left(c_{w}-c_{0}\right) C_{7}(X, Y), \\
X=\left[\int_{0}^{x} u_{7}(p) d p\right] X_{7}^{-1}, \\
Y=y u_{7}(x)\left(\kappa X_{7}\right)^{-\frac{1}{2}}, \\
X_{7}=\int_{0}^{x_{7}} u_{7}(p) d p .
\end{gathered}
$$

These transformations hold for $0 \leq x \leq x_{7}$. For concentration profile number 9 , we introduce [20]:

$$
\begin{gathered}
c_{9}=c_{0}+\left(c_{w}-c_{0}\right) C_{9}(Q, N), \\
Q=\left[\int_{0}^{q} \alpha_{9}^{\frac{1}{2}}(p) d p\right] Q_{9}^{-1}, \\
N=n \alpha_{9}^{\frac{1}{2}}(q)\left(\kappa Q_{9}\right)^{-\frac{1}{3}}, \\
Q_{9}=\int_{0}^{q_{9}} \alpha_{9}^{\frac{1}{2}}(p) d p .
\end{gathered}
$$

These transformations hold for $0 \leq q \leq q_{9}$. For the concentration layers along the edge-hole boundary, similar transformations are used. These transformations can be derived from (3.16) to (3.19) by replacing of the parameters $q, n, Q$ and $N$ by $\phi, \theta, \Phi$ and $\Theta$.

With the transformations (3.12) to (3.15), Eq. (2.4) with boundary conditions (2.5) reduces to

$$
\frac{\partial C_{7}}{\partial X}=\frac{\partial^{2} C_{7}}{\partial Y^{2}}, \quad 0 \leq X \leq 1,-\infty \leq Y \leq \infty
$$

with boundary conditions

$$
\frac{\partial C_{7}}{\partial Y} \rightarrow 0 \text { if } Y \rightarrow-\infty \text { and } Y \rightarrow \infty .
$$

Since the value of the diffusion coefficient $\kappa$ in (3.14) is very small, the range of $Y$ is large and $Y= \pm \infty$ is a good approximation for the edge of the boundary layer. 
With transformations (3.16) to (3.19), Eq. (2.6) with boundary conditions (2.7) reduces to

$$
N \frac{\partial C_{9}}{\partial Q}=\frac{\partial^{2} C_{9}}{\partial N^{2}}, 0 \leq Q \leq 1,0 \leq N \leq \infty,
$$

with boundary conditions

$$
\frac{\partial C_{9}}{\partial N}=0 \text { if } N=0 \text { and } N \rightarrow \infty .
$$

For boundary layer 2 an equation similar to (3.22) can be obtained, but the boundary condition $(2.8)$ is

$$
C_{2}=1 \text { if } \Theta=0 .
$$

Similar equations can be derived for the other boundary layers.

We can use the scaled quantities to derive matching conditions for the concentration profiles at the boundary interfaces. With (3.12) to (3.19), the relations at the boundary interfaces (2.9) and (2.11) can be rewritten as

$$
C_{2}\left(1, \omega_{7,2} N^{2}\right)=C_{7}(0, N) \text { and } C_{6}\left(1, \zeta_{6,5} N\right)=C_{5}(0, N)
$$

where

$$
\omega_{7,2}=\frac{1}{2} \frac{\left(\kappa Q_{2}\right)^{\frac{2}{3}}}{\left(\kappa X_{7}\right)^{\frac{1}{2}}} \text { and } \zeta_{6,5}=\frac{\left(Q_{5}\right)^{\frac{2}{3}}}{\left(\Phi_{6}\right)^{\frac{2}{3}}} .
$$

For all other boundary interfaces different $\omega_{i, j}$ or $\zeta_{i, j}$ can be defined. The boundary condition for the concentration profile at the beginning of the first boundary layer is

$$
C_{1}(0, Y)=0 \text { if }-\infty \leq Y \leq 0 .
$$

The only parameters in the systems of differential equations are $\omega$ and $\zeta$. These parameters take the influence of the geometry and velocity field into account.

For all boundary layers, an integral description of the solution can be constructed. The derivation of these solutions is shown in Appendix A. For the boundary layers along free streamlines, the representation of the concentration is given by

$$
C_{i}(X, Y)=\frac{1}{2 \pi^{\frac{1}{2}} X^{\frac{1}{2}}} \int_{-\infty}^{\infty} C_{i}(0, P) e^{-\frac{(P-Y)^{2}}{4 X}} d P .
$$

For $i=1$, corresponding to the upper free boundary layer, the initial profile for $-\infty \leq Y \leq 0$ is equal to zero. For this concentration profile, the interval for integration can be reduced to $[0, \infty>$. For the boundary layers $4,5,9$ and 10 along the masks, the concentration profile is given by

$$
C_{i}(Q, N)=\frac{1}{3 Q} \int_{0}^{\infty} N^{\frac{1}{2}} P^{\frac{3}{2}} e^{-\frac{N^{3}+P^{3}}{9 Q}} I_{-\frac{1}{3}}\left(\frac{2}{9} \frac{N^{\frac{3}{2}} P^{\frac{3}{2}}}{Q}\right) C_{i}(0, P) d P
$$


where $I_{-\frac{1}{3}}(P)$ is a modified Bessel function. For the boundary layers 2, 6 and 8 along the edge-hole boundary, the integral solution is

$$
C_{i}(\Phi, \Theta)=\frac{\Gamma\left(\frac{1}{3}, \frac{\Theta^{3}}{9 \Phi}\right)}{\Gamma\left(\frac{1}{3}\right)}+\frac{1}{3 \Phi} \int_{0}^{\infty} \Theta^{\frac{1}{2}} P^{\frac{3}{2}} e^{-\frac{\Theta^{3}+P^{3}}{9 \Phi}} I_{\frac{1}{3}}\left(\frac{2}{9} \frac{\Theta^{\frac{3}{2}} P^{\frac{3}{2}}}{\Phi}\right) C_{i}(0, P) d P,
$$

where $\Gamma(a, b)=\int_{b}^{\infty} t^{a-1} e^{-t} d t$ is the incomplete Gamma function. By using the matching conditions at the interfaces of the boundary layers like (3.25), all integral representations are coupled to form a system of equations for the unknown profiles $C_{i}(0, P)$.

It is not possible to find analytical solutions to these equations. Therefore, we will compute the solutions numerically. To this end the region of integration is separated in two parts: a finite interval, $\left[-P_{m}, P_{m}\right]$ for the free concentration layers and $\left[0, P_{m}\right]$ for the other boundary layers, and infinite intervals, $\left.<-\infty,-P_{m}\right]$ and $\left[P_{m}, \infty>\right.$ for the free concentration layers and one interval $\left[P_{m}, \infty>\right.$ for the other boundary layers. The assumption in the previous subsection was that the concentration only varies in a small region of the boundary layers. We choose the value of $P_{m}$ in such a way that outside of the interval $\left[-P_{m}, P_{m}\right]$ for free boundary layers and $\left[0, P_{m}\right]$ for the other boundary layers the concentration is approximately constant. Only one value of $P_{m}$ has to be chosen. From the relations between the profiles at the beginning and at the end of the boundary layers such as (3.25), the values of $P_{m}$ can be calculated for all boundary layers.

Because there are three eddies inside the etch hole, we have three constant concentration levels. The value in the central eddy is called $C_{c}$, the value in the right eddy $C_{r}$, and the value in the left eddy $C_{l}$. For the different boundary layers the integrals over the infinite intervals can be determined analytically. For the free boundary layers, we obtain the following integral expression from (3.28)

$$
\begin{aligned}
C_{i}(X, Y)=\frac{1}{2 \pi^{\frac{1}{2}} X^{\frac{1}{2}}} \int_{-P_{m}}^{P_{m}} & C_{i}(0, P) e^{-\frac{(P-Y)^{2}}{4 X}} d P \\
& +\frac{1}{2} C_{i}\left(X, P_{m}\right) \operatorname{erfc}\left(\frac{P_{m}-Y}{2 \sqrt{X}}\right)+\frac{1}{2} C_{i}\left(X,-P_{m}\right) \operatorname{erfc}\left(\frac{P_{m}+Y}{2 \sqrt{X}}\right) .
\end{aligned}
$$

For the boundary layers along the mask, the integral expression can be rewritten as [10]

$$
\begin{aligned}
C_{i}(Q, N)=C_{i}\left(Q, P_{m}\right)+ \\
\frac{1}{3 Q} \int_{0}^{P_{m}} N^{\frac{1}{2}} P^{\frac{3}{2}} e^{-\frac{N^{3}+P^{3}}{9 Q}} I_{-\frac{1}{3}}\left(\frac{2}{9} \frac{N^{\frac{3}{2}} P^{\frac{3}{2}}}{Q}\right)\left[C_{i}(0, P)-C_{i}\left(0, P_{m}\right)\right] d P .
\end{aligned}
$$

A concentration profile in the boundary layers along the etch hole has the following integral representation [10]

$$
\begin{aligned}
& C_{i}(\Phi, \Theta)=C_{i}\left(\Phi, P_{m}\right)+ {\left[1-C_{i}\left(\Phi, P_{m}\right)\right] \frac{\Gamma\left(\frac{1}{3}, \frac{\Theta^{3}}{9 \Phi}\right)}{\Gamma\left(\frac{1}{3}\right)}+} \\
& \frac{1}{3 \Phi} \int_{0}^{P_{m}} \Theta^{\frac{1}{2}} P^{\frac{3}{2}} e^{-\frac{\Theta^{3}+P^{3}}{9 \Phi}} I_{\frac{1}{3}}\left(\frac{2}{9} \frac{\Theta^{\frac{3}{2}} P^{\frac{3}{2}}}{\Phi}\right)\left[C_{i}(0, P)-C_{i}\left(0, P_{m}\right)\right] d P .
\end{aligned}
$$


As results only finite integration intervals remain in the system of equations, and this simplifies the numerical computations.

The concentration is calculated in order to construct a numerical simulation of wet-chemical etching. The dissolution of material into the fluid depends only on the concentration derivative normal to the etch-hole boundary. From the description of the concentration along the etchhole boundary layers this derivative can be found by differentiation with respect to $\Theta$. As an example, for boundary layer 2 this results in

$$
\left.\frac{\partial C_{2}}{\partial \Theta}\right|_{\Theta=0}=\left(C_{c}-1\right) \frac{3^{\frac{1}{3}}}{\Gamma\left(\frac{1}{3}\right)} \Phi^{-\frac{1}{3}}+\frac{3^{-\frac{2}{3}}}{\Gamma\left(\frac{1}{3}\right)} \Phi^{-\frac{4}{3}} \int_{0}^{P_{m}} P^{2} e^{-\frac{P^{3}}{9 S}} C_{2}(0, P) d P .
$$

For the other etch-hole boundary layers similar expressions can easily be derived from (3.34).

\section{NUMERICAL IMPLEMENTATION}

In this section, the numerical computation of the concentration profiles is described. In a similar way as described in Eq. (3.25) 12 equations for 12 unknown profiles can be derived. In the previous section it was described how the integrals are reduced to integrals over a finite interval. The only remaining problem is the discretization of the integral relations.

For a given value of $P_{m}$, the interval $\left[0, P_{m}\right]$ is discretized into a uniform grid. The kernels of the integrals are represented point-wise in the center of the intervals. Grid refinement showed that such an approximation is sufficiently accurate.

The coupling of the integral relations is done in a special way. When we consider the stream function values in the different boundary layers (2.10), it can be seen that the stream function profile is quadratic in the free boundary layers, and linear in the boundary layers along the mask and along the wall. Therefore, the profiles at the beginning and at the end of the free boundary layers are replaced by the corresponding profiles at the end or, respectively, at the beginning of the profiles along the mask or the wall.

We give here an example of this substitution. The free boundary layer 3 is connected to four other boundary layers. The end of this layer is the beginning of the boundary layers 2 and 6. To obtain an equation for the profile at the beginning of boundary layer 6 , we substitute $C_{3}\left(1,-\omega_{3,6} N^{2}\right)=C_{6}(0, N)$ in the description of $C_{3}$. For the profile at the beginning of $C_{3}$ we substitute $C_{3}\left(0,-\omega_{3,5} N^{2}\right)=C_{5}(1, N)$ and $C_{3}\left(0, \omega_{3,4} N^{2}\right)=C_{4}(1, N)$ in the description of $C_{3}$, which results in

$$
\begin{aligned}
C_{6}(0, \Theta)= & \frac{\omega_{3,4}}{\pi^{\frac{1}{2}}} \int_{0}^{P_{m}} P e^{-\frac{\left(\omega_{3,4} P^{2}+\omega_{3,6} \Theta^{2}\right)^{2}}{4}} C_{4}(1, P) d P+\frac{C_{c}}{2} \operatorname{erfc}\left(\frac{1}{2}\left[\omega_{3,4} P_{m}^{2}+\omega_{3,6} \Theta^{2}\right]\right) \\
& +\frac{\omega_{3,5}}{\pi^{\frac{1}{2}}} \int_{0}^{P_{m}} P e^{-\frac{\left(-\omega_{3,5} P^{2}+\omega_{3,6} \Theta^{2}\right)^{2}}{4}} C_{5}(1, P) d P+\frac{C_{r}}{2} \operatorname{erfc}\left(\frac{1}{2}\left[\omega_{3,5} P_{m}^{2}-\omega_{3,6} \Theta^{2}\right]\right),
\end{aligned}
$$

where the values of $\omega$ are computed as in (3.26).

As another result of the substitution of the profiles at the beginning and at the end of the free boundary layer number 3 , the integral formulation for the coupling of $C_{4}$ and $C_{3}$ becomes:

$$
C_{4}(1, N)=C_{c}+\frac{1}{3} \int_{0}^{P_{m}} N^{\frac{1}{2}} P^{\frac{3}{2}} e^{-\frac{N^{3}+P^{3}}{9}} I_{-\frac{1}{3}}\left(\frac{2}{9} N^{\frac{3}{2}} P^{\frac{3}{2}}\right)\left[C_{4}(0, P)-C_{c}\right] d P .
$$


Other integral relations can be derived in a similar way. In Section 7, results from the described boundary-layer method are presented.

\section{Finite-volume method}

A finite-volume method was implemented for validation of the assumptions mentioned in subsection 2.2. This validation is performed for an etch-hole geometry in which the undercut effect shown in Fig. 3 is disregarded. In this section, we describe the finite volume implementation.

We apply the finite-volume method to the stationary convection-diffusion equation (2.2) with a known velocity field. Another way to describe (2.2) is given by

$$
\operatorname{div}(\boldsymbol{u} \cdot c-D \operatorname{grad} c)=0 .
$$

We integrate (5.37) over a control volume $k$, and apply the Gauss theorem which results in:

$$
\int_{S_{k}}[\boldsymbol{u} \cdot c-D \operatorname{grad} c] \cdot \boldsymbol{n d} S_{k}=0,
$$

where $S_{k}$ is the surface of the control volume $k$ and $\boldsymbol{n}$ is the outward unit vector normal at $S_{k}$. In the two-dimensional application considered in this paper, this surface consists of the four boundary segments surrounding point $(i, j)$.

The finite-volume method consists of the discretization of (5.38) for each volume part of the domain.

\subsection{Discretization}

We now describe how the domain is divided into control volumes and the discretization used for the convective and diffusive terms. It is not necessary to make a finite-volume discretization of the complete geometry. In the case of a uniform flow along the cavity an eddy will arise in the interior. We only make a structured grid for the boundary layer near the cavity wall and along the dividing streamline that separates the eddy from the outer flow. In Fig. 7 this grid is given for a certain mesh size. At the center of this grid, which is the center of the eddy, we assume a constant concentration. The boundary condition for the surrounding grid cells is that the normal derivative pointing into the center is equal to zero. The control volumes are defined as in Fig. 8. The surface $A B C D$ is the volume to which (5.38) is applied.

For the diffusion terms, we need to discretize $\frac{\partial c}{\partial n}$. The discretization of this quantity on the volume boundary $A B$ is approximated by

$$
\frac{\partial c}{\partial n}=1 / 2\left[\left.\frac{\partial c}{\partial x}\right|_{A} n_{1}+\left.\frac{\partial c}{\partial y}\right|_{A} n_{2}+\left.\frac{\partial c}{\partial x}\right|_{B} n_{1}+\left.\frac{\partial c}{\partial x}\right|_{B} n_{2}\right],
$$

where $\left.\frac{\partial c}{\partial x}\right|_{A}$ is computed as

$$
\left.\frac{\partial c}{\partial x}\right|_{A}=\frac{1}{S_{A}} \int_{\partial S_{A}} c n_{x} \mathrm{~d} l_{S_{A}}
$$


with $S_{A}$ the surface between the points $(i, j-1),(i+1, j-1),(i+1, j)$ and $(i, j)$. The convective terms are discretized by a second-order upwind method.

\subsection{Boundary conditions}

At the etch-hole boundary, a Dirichlet boundary condition is enforced. If the line $j=1 / 2$ coincides with the boundary, this condition is implemented in the following way at $c_{i, 1}$. We introduce a virtual point $c_{i, 0}$ which is at the same distance on the opposite side of the boundary. For the discretizations where this virtual point is taken into account, we substitute

$$
c_{i, 0}=2 c_{w}-c_{i, 1}
$$

where $c_{w}$ is the known concentration at the boundary.

At the mask, a Neumann boundary condition is applied. Because the normal derivatives along the boundaries of the control volumes are specified, it is easy to implement the Neumann condition

$$
\frac{\partial c}{\partial n}=0
$$

Above the etch-hole, the boundary conditions depend on the direction of the fluid flow. In the fluid that flows in the direction of the cavity, the concentration of the dissolved material is equal to a value $c_{0}$. In the fluid that flows out of the cavity, a boundary condition is not required.

The discretization results in a linear system of equations. A standard way to solve the linear system is to use a symmetric Gauss-Seidel algorithm [3]. However, due to the second-order upwind discretization, the symmetric Gauss-Seidel algorithm converges very slowly. Therefore, the system is solved first for a first-order upwind discretization. The solution of this system is used as an initial solution for the second-order linear system. In this way, a second-order accurate solution can be computed.

\section{VALidation}

The convection-diffusion equation is solved by means of the finite-volume method. The only assumption made is that the concentration near the center of the eddy is constant. Therefore, a grid is not necessary there (see Fig. 7). We have implemented this assumption by setting the normal concentration derivative at the inner boundary of the grid equal to zero. As mentioned in the introduction, the method is especially applicable for situations with high Péclet numbers. The characteristic velocity is evaluated at the centerline near the mouth of the cavity and the distance between the masks is taken as the characteristic lengthscale. The scaled diffusion coefficient in $(2.2)$ is taken equal to $3.1 \times 10^{-6}$, which corresponds to a Péclet number of about $P e \approx 90,000$. The scaled concentration at the etch-hole boundary $C_{w}$ is taken equal to unity. In Fig. 9, the finite-volume solution of the convection-diffusion equation is shown. In this section we will check the assumptions made in subsection 2.2 by comparison with the finite-volume results. 
To give an indication of the time needed for the boundary-layer method the unknown profiles were computed in less than one second on a single R10000 processor, while it took on the order of minutes to compute a finite-volume solution of the same accuracy.

To confirm that the concentration near the center of the eddy is constant, we inspect the concentration as computed with the finite-volume method at the grid points near the center. The scaled concentration values at these points vary between 0.33 and 0.3314 which is a maximum difference of less than $0.5 \%$.

To confirm that the diffusion in the stream-wise direction is negligible in comparison with the diffusion in the normal direction, we computed the second derivatives from the solution of the finite-volume method. The second derivative in the normal direction is of $O\left(10^{2}\right)$ along the etch-hole boundary, while the second derivative in tangential direction is of $O(1)$. However, near the beginning and the end of the free streamline, where the flow makes a sharp bend, these quantities are of the same order of magnitude.

To validate the assumptions about the linearity of the velocity profiles near the etch-hole boundary, we plotted these profiles at three places. In Fig. 10, profiles of the streamwise velocity (solid lines) as well as the scaled concentration (dashed lines) are given as functions of the distance to the etch-hole boundary. Line (a) is near the bottom of the etch-hole, line (c) is near the region where in the boundary-layer method the boundary layers are matched and (b) lies in between. We can conclude that the linearity assumption holds in the region where the concentration varies.

In Fig. 11 the velocity profile at the midpoint of the free streamline is plotted. The dashed line shows the scaled concentration profile at this location. In this figure, it can be seen that the velocity is fairly constant in the region where the concentration varies.

The concentration is computed in order to construct a numerical simulation of wet-chemical etching. The dissolution of material into the fluid depends only on the concentration derivative normal to the etch-hole boundary. Therefore, the most important validation arises from the comparison of this derivative. In Fig. 12, the concentration derivative normal to the etch-hole boundary is shown for both methods. Near the bottom of the etch-hole the difference is less than $1 \%$, near the beginning and the end of the free stream-line the difference is $1-3 \%$. These small differences justify the extension of the boundary-layer method to a system with overhanging mask edges. In a separate paper, the extended system will be included in a numerical simulation. In such a simulation, the numerical errors can cause an over- or underestimation of the displacement of the etch-hole boundary. In the next section we show that the algorithm described in this paper will smooth out this error in the subsequent time steps.

\section{Results}

In this section, we present some results of computations in etch-hole geometries. We first use the geometry as shown in Fig. 4, with 10 boundary layers. The fluid-flow and shear-stress are computed with the boundary-element method as described in [6]. In Fig. 13 concentration profiles are given as functions of a scaled normal distance. Profiles 2, 4, 5, 6, 8, 9, and 10 are at the beginning of the corresponding boundary layers. Profiles $2^{*}, 4^{*}, 5^{*}, 8^{*}$ and $10^{*}$ are at the end of the corresponding boundary layers. These profiles are equal to the profiles at the beginning of boundary layers 1,3 and 7 . 
From Fig. 13 it can be concluded that the concentration reaches different asymptotic levels in the different eddies. In the central eddy this concentration level is low because this eddy is attached to the fluid outside by the dividing stream-line. The asymptotic concentration level in the center of the right eddy is lower than the asymptotic concentration level in the center of the left eddy. To explain this, we recall that the fluid flows in a clockwise direction in the central eddy. The fluid becomes more and more 'polluted' with dissolved material. Therefore, the concentration of the fluid will be higher at the left side of the etch-hole, which results in a higher asymptotic concentration level in the left eddy. A concentration profile with a scaled asymptotic concentration equal to zero is not plotted in this figure. Such profiles are present at the beginning and at the end of boundary layer 1, but are not included in the matching procedures. The profile at the beginning of this boundary layer for $y \leq 0$ is equal to zero, the profile at the end can be computed by (3.31).

Another result of the computation is the distribution of the normal derivative of the concentration at the etch-hole boundary. With this normal derivative, the movement of the etch-hole boundary can be computed. In Fig. 14 the derivative is plotted at the boundary. A large derivative will result in a high etch speed. From this picture, it can be seen that the etch-hole boundary will become asymmetric as a result of the fluid flow. The second line plotted in Fig. 14 is the normal concentration derivative when locally a small hole is included in the geometry. The geometry and the modified geometry are also shown in the figure. From the corresponding plot of the normal derivative, it can be concluded that such holes are smoothed out by the etching process. As a result of such a hole, the etch rate at the bottom of the hole decreases and the etch rate increases at both sides.

As a second example of a concentration computation in an etch-hole geometry, we consider the concentration for the initial geometry of the etching process, shown in Fig. 15. At the beginning of the etching process only small vortices are present at both sides near the masks and the central eddy present in Fig. 3 has not been formed yet. We apply the boundary-layer method to this geometry. Fig. 16 shows the numbering of the boundary layers. We take the ratio of depth and width of the cavity equal to 1:4. In Fig. 17, the concentration profiles at the beginning or at the end (with an asterix) of the boundary layers are given as a function of a scaled normal distance computed for $P e=90,000$. Here, a theory similar to that stated above about the asymptotic concentration levels holds.

In Fig. 18 the normal derivate of the concentration is plotted along the bottom of the etch hole for different values of the Péclet number. These Péclet number values are chosen to make the results comparable to those plotted in [23]. Occhialini et al. used a boundary-element method for the flow computation, and an orthogonal-collocation method to solve the convection-diffusion equation. The results are very much alike. Because the boundary-layer method is only applicable at large Péclet numbers we computed the derivatives only for $P e=3000,5000,10000$ and 15000 respectively. As mentioned in [23] the concentration derivative is low near the corners as a result of the secondary corner eddies predicted by Moffatt [21]. However, the boundary-layer method shows mild fluctuations near these corners. The fluctuations appear as a result of the secondary eddies, which are not accounted for in the boundary-layer model. This sensitivity problem will be tackled in a future paper.

For both etch-hole geometries, one can notice the difference in normal concentration derivative 
at the stagnation and separation point. At the stagnation point the fluid flows toward the boundary and the concentration gradient is smooth. Near the end of the boundary layers, the fluid flows away from the separation point. Here, the normal concentration derivative has a local minimum. As a result of this local minimum, etching will proceed slowly at the separation point, and this will result in an non-smooth etch-hole boundary. However, as the depth of the etch-hole increases, the stagnation and separation points will move towards each other, and this finally results in a central eddy.

\section{Concluding REMARKS}

In this paper we have defined a semi-analytical way for the computation of the concentration in an etch-hole configuration. Using a number of assumptions, we have constructed analytical descriptions in different sub-domains. By coupling these sub-domains, the concentration and its normal derivative at the etch-hole boundary can be computed. The advantage of the boundarylayer method compared to spectral-element or finite-element methods is that a computational grid for the interior is not required, and only the boundary and the dividing streamlines inside the domain need to be described. In this way, the etching process can be simulated with little computational power and memory.

Although the validation showed that not all assumptions are valid throughout the entire domain, the concentration gradient can be computed with an accuracy that is sufficiently good to justify the use of this method in a numerical etch simulation. The initial etch-hole geometry showed good qualitative agreement with earlier fully numerical results reported in [23]. Only near the corners the boundary-layer method shows sensitivity to the smaller corner eddies. This will be considered in a future paper. A modified version of the method presented can be applied to axisymmetric three-dimensional cases too, such as described in [18].

\section{Acknowledgement}

This work was supported by STW, the Netherlands Technology Foundation, under project TWI44.3286. The authors thank Professor P.J. Zandbergen and Professor C. Pozrikidis for stimulating discussions and recommendations.

\section{REFERENCES}

1. M. Abramowitz and I.A. Stegun, Handbook of mathematical functions. Dover Publications, New York ,1970.

2. R.C. Alkire, H. Deligianni and J.-B. Ju, Effect of fluid flow on convective transport in small cavities. J. Elchem. Soc., 1990, 137, 818-824.

3. R. van Buuren, J.G.M. Kuerten and B.J. Geurts, Instabilities of stationary inviscid compressible flow around an airfoil. J. Comp. Phys., 1997, 138, 520-539.

4. G. Camera-Roda, C. Boi, A. Saavedra and G. C. Sarti, Heat and mass transfer boundary layers in radial creeping flow. Int. J. Heat Mass Transfer, 1994, 37, 2145-2153. 
5. C.H. Driesen, J.G.M. Kuerten and M. Streng, Low-Reynolds-number flow over partially covered cavities. J. Eng. Math., 1998, 34, 5-24.

6. C.H. Driesen and J.G.M. Kuerten, An accurate boundary-element method for Stokes flow in partially covered cavities. to be published.

7. M. Georgiadou and R.C. Alkire, Anisotropic chemical etching of copper foil. I. Electrochemical studies in acidic $\mathrm{CuCl}_{2}$ solutions. J. Elchem. Soc., 1993, 140, 1340-1347.

8. M. Georgiadou and R.C. Alkire, Anisotropic chemical etching of copper foil. II. Experimental studies on shape evolution. J. Elchem. Soc. 1993, 140, 1348-1355.

9. M. Georgiadou and R.C. Alkire, Anisotropic chemical pattern etching of copper foil. III Mathematical model. J. Elchem. Soc., 1994, 141, 679-689.

10. I.S. Gradshteyn and I.W. Ryzhik, Table of integrals, series and products. Academic Press, New York, 1980.

11. J.N. Harb and R.C. Alkire, Transport and reaction during pitting corrosion of Ni in $0.5 \mathrm{M} \mathrm{NaCl.} \mathrm{J.}$ Elchem. Soc., 1991, 138, 3568-3575.

12. K.G. Jordan and C.W. Tobias, Simulation of the role of convection in electro-deposition into microscopic trenches. J. Elchem. Soc., 1997, 138, 1933-1939.

13. K. Kondo, K. Fukui, K. Uno and K. Shinohara, Shape evolution of electro-deposited copper bumps. J. Elchem. Soc., 1996, 143, 1880-1886.

14. H.K. Kuiken, Heat or mass transfer from an open cavity. J. Eng. Math., 1978, 40, 129-155.

15. H.K. Kuiken, J.J. Kelly and P.H.L. Notton, Etching profiles at resist edges. J. Elchem. Soc., 1986, 133, 1217-1226.

16. H.K. Kuiken, Etching: a two-dimensional mathematical approach. Proc. R. Soc. Lond. A, 1984, 392, 199-225.

17. H.K. Kuiken, Etching through a slit. Proc. R. Soc. Lond. A, 1984, 396, 95-117.

18. H.K. Kuiken, A free-convection boundary-layer model for the centrifugal etching of an axisymmetric cavity. Journal of Engineering Mathematics, 1998, 34, 181-200.

19. R.D. Lazarov, I.D. Mishev and P.S. Vasselevski, Finite volume methods for convection-diffusion problems. SIAM J. Numer. Anal., 1996, 33, 31-55.

20. M.J. Lighthill, Contributions to the theory of heat transfer through a laminar boundary layer. Proc. R. Soc. London A, 1950, 202, 369-377.

21. H.K. Moffatt, Viscous and resistive eddies near a sharp corner. J. Fluid. Mech. , 1964, 18, 1-18.

22. F. Oberhettinger and L. Badii, Tables of Laplace Transforms. Springer, Berlin, 1973.

23. J.M. Occhialini and J.J.L. Higdon, Convective mass transport from rectangular cavities in viscous flow. J. Elchem. Soc., 1992, 139, 2845-2855.

24. C.B. Shin and D.J. Economou, Effect of transport and reaction on the shape evolution of cavities during wet chemical etching. J. Elchem. Soc., 1989, 136, 1997-2004.

25. C.B. Shin and D.J. Economou, Forced and natural convection effects on the shape evolution of cavities during wet chemical etching. J. Elchem. Soc., 1991, 138, 527-538.

26. C.B. Shin and D.J. Economou, Mass transfer by natural and forced convection in open cavities. Int. J. Heat Mass Transfer, 1990, 33, 2191-2205.

27. N.M. Temme, Speciale functies in de mathematische fysica. Epsilon Publications, Utrecht, The Netherlands, 1990, 132-149.

28. C. Vuik and C. Cuvelier, Numerical solution of an etching problem. J. Comp. Phys., 1985, 59, 247-263. 


\section{APPENDIX}

In this Appendix, we explain how the integral formulations for the concentration profiles presented in Section 2 are derived. The integral formulations for the concentration profiles in the layers along the free streamlines follow from the differential equation by separation of variables. These solutions do not need further explanation. The other two integral formulations are discussed here. We consider the scaled problems

$$
N \frac{\partial C}{\partial Q}=\frac{\partial^{2} C}{\partial N^{2}}, \quad 0 \leq Q \leq 1,0 \leq N<\infty
$$

with boundary conditions

$$
\frac{\partial C}{\partial N}=0 \text { if } N=0 \text { and } N \rightarrow \infty
$$

For the boundary layers along the etch-hole boundary, an equation similar to (8.43) can be obtained by substitution of the local variables as mentioned in section 2 , but with different boundary conditions. We will derive an integral-equation solution for (8.43). The boundary condition at $Q=0$ is a function of $N$ alone

$$
C(0, N)=F(N) .
$$

We can start this derivation by using a special form of this function $F(N)$, the Dirac-delta function.

$$
F(N)=\delta(N-B)
$$

where $B$ is an arbitrary point, $B \geq 0$. We apply a Laplace transformation

$$
\tilde{C}(s, N)=\int_{0}^{\infty} e^{-s Q} C(Q, N) d Q
$$

to (8.43) with boundary conditions (8.44), which gives

$$
\frac{\partial^{2} \tilde{C}}{\partial N^{2}}=N[s \tilde{C}-\delta(N-B)]
$$

with boundary conditions

$$
\frac{\partial \tilde{C}}{\partial N}=0 \text { if } N=0 \text { and } N \rightarrow \infty
$$

Since $\delta(N-B)$ is equal to zero for any $N$ which is not equal to $B,(8.48)$ can be rewritten as

$$
\frac{\partial^{2} \tilde{C}}{\partial N^{2}}=N s \tilde{C}, \text { if } N \neq B
$$


The effect of the delta function on the solution can be found by integration of (8.48) around the point $B$, leading to

$$
\left.\frac{\partial \tilde{C}}{\partial N}\right|_{N=B-0} ^{N=B+0}=-B
$$

When a substitution $z=N s^{\frac{1}{3}}$ is applied to (8.50) we obtain

$$
\frac{\partial^{2} \tilde{C}^{*}}{\partial z^{2}}=z \tilde{C}^{*}
$$

This equation has a general solution which can be found in [1]. Using (8.51) and the boundary conditions the solution is given by

$$
\tilde{C}(s, N)=\frac{2}{3} N^{\frac{1}{2}} B^{\frac{3}{2}} K_{\frac{1}{3}}\left(\frac{2}{3} s^{\frac{1}{2}} B^{\frac{3}{2}}\right) I_{-\frac{2}{3}}\left(\frac{2}{3} s^{\frac{1}{2}} N^{\frac{3}{2}}\right), \quad(0 \leq N<B),
$$

and

$$
\tilde{C}(s, N)=\frac{2}{3} N^{\frac{1}{2}} B^{\frac{3}{2}} K_{\frac{1}{3}}\left(\frac{2}{3} s^{\frac{1}{2}} N^{\frac{3}{2}}\right) I_{-\frac{2}{3}}\left(\frac{2}{3} s^{\frac{1}{2}} B^{\frac{3}{2}}\right), \quad(N \geq B),
$$

where $K_{\frac{1}{3}}$ and $I_{-\frac{1}{3}}$ are modified Bessel functions. The Laplace inverses of these functions can be found in [22] which gives for the concentration as a function of a point source at point $B$

$$
C(Q, N)=\frac{1}{3} Q^{-1} N^{\frac{1}{2}} B^{\frac{3}{2}} e^{-\frac{B^{3}+N^{3}}{9 Q}} I_{-\frac{1}{3}}\left(\frac{2}{9} \frac{N^{\frac{1}{2}} B^{\frac{3}{2}}}{Q}\right) .
$$

Using convolution, the integral formulation for the concentration is obtained as

$$
C(Q, N)=\frac{1}{3 Q} \int_{0}^{\infty} N^{\frac{1}{2}} P^{\frac{3}{2}} e^{-\frac{N^{3}+P^{3}}{9 Q}} I_{-\frac{1}{3}}\left(\frac{2}{9} \frac{N^{\frac{3}{2}} P^{\frac{3}{2}}}{Q}\right) F(P) d P .
$$

The solution procedure is similar to the solution procedure of the differential equation for the concentration profiles along the etch-hole boundary. 


\section{Figure captions}

Figure 1: Initial configuration for etching a groove in a rotating disk.

Figure 2: The wet-chemical etching process.

Figure 3: Iso lines of the stream function for a typical etch-hole geometry.

Figure 4: Definition of boundary layers.

Figure 5: Definition of local coordinates in boundary layers.

Figure 6: Coupling of stream-function values.

Figure 7: Computational grid for the finite-volume method.

Figure 8: Control volume for grid point $(i, j)$. The arrow denotes the normal on the edge.

Figure 9: Iso lines of the scaled concentration computed with the finite-volume method.

Figure 10: Stream-wise velocity as a function of the normal distance to the etch-hole boundary (solid lines) in comparison with the variation of the scaled concentration (dashed lines).

Figure 11: Stream-wise velocity (solid line) as a function of $y$ in the region where the scaled concentration (dashed line) varies.

Figure 12: Normal derivatives of the concentration at the etch-hole boundary computed with the finite-volume and the boundary-layer method.

Figure 13: Scaled concentration profiles for different boundary layers.

Figure 14: Normal derivatives of the concentration at the etch-hole boundary for a smooth geometry and a geometry with a small local hole.

Figure 15: Iso lines of the streamfunction for an initial etch-hole geometry.

Figure 16: Definition of boundary layers.

Figure 17: Scaled concentration profiles for different boundary layers.

Figure 18: Normal derivatives of the concentration at the etch-hole boundary for $P e=3000$ (a), 5000 (b), 10000 (c) and 15000 (d). 


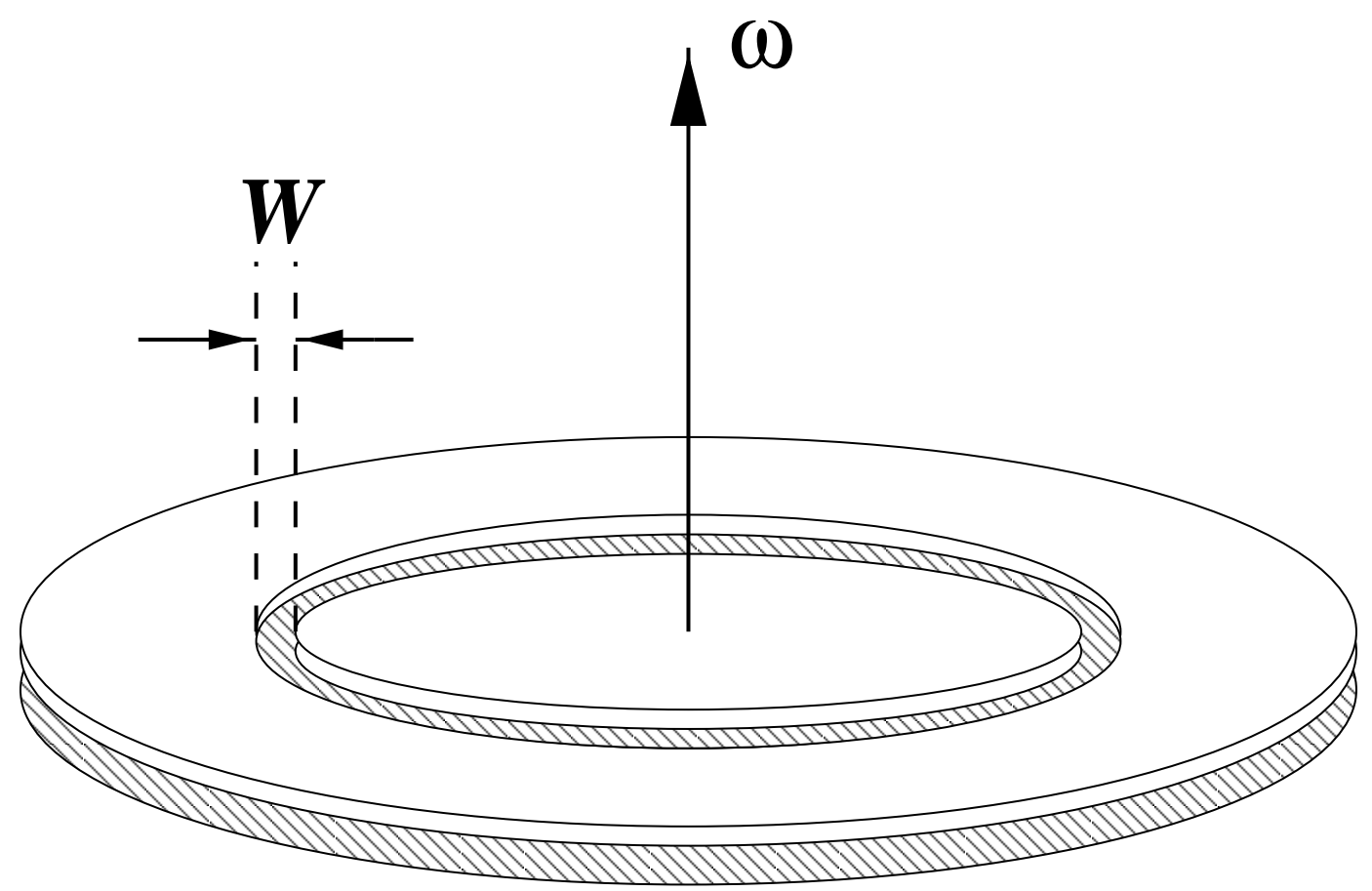


FIGURE 2
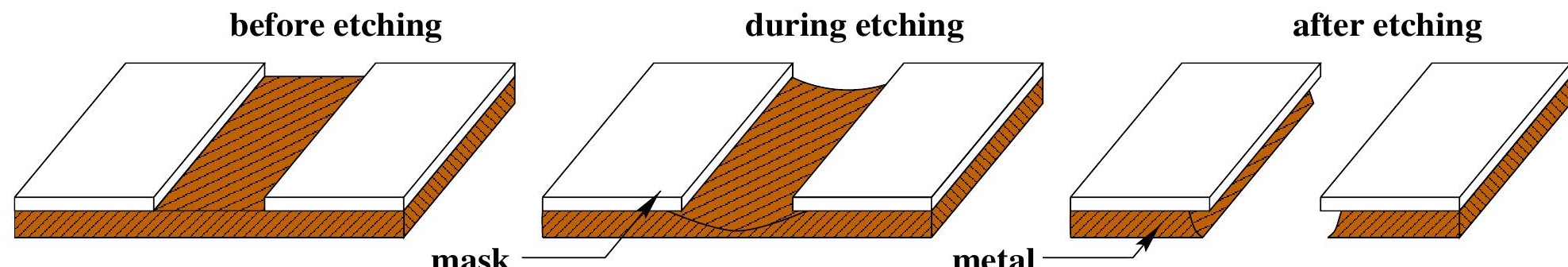
Figure 3

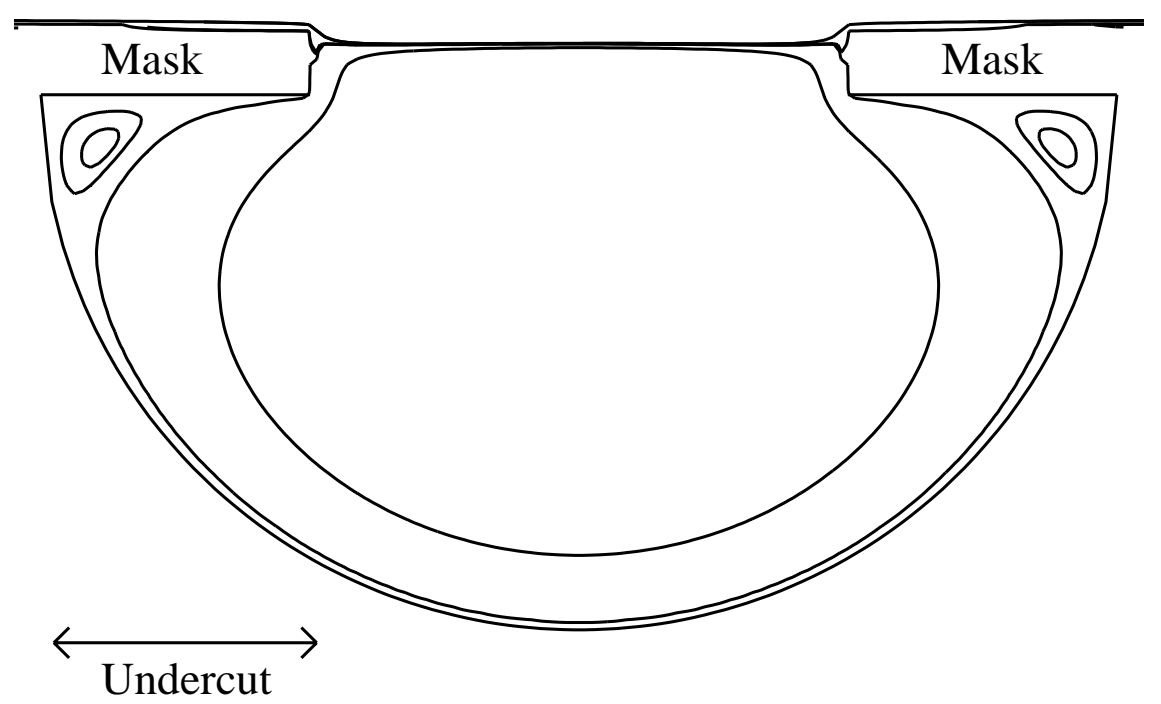


Figure 4

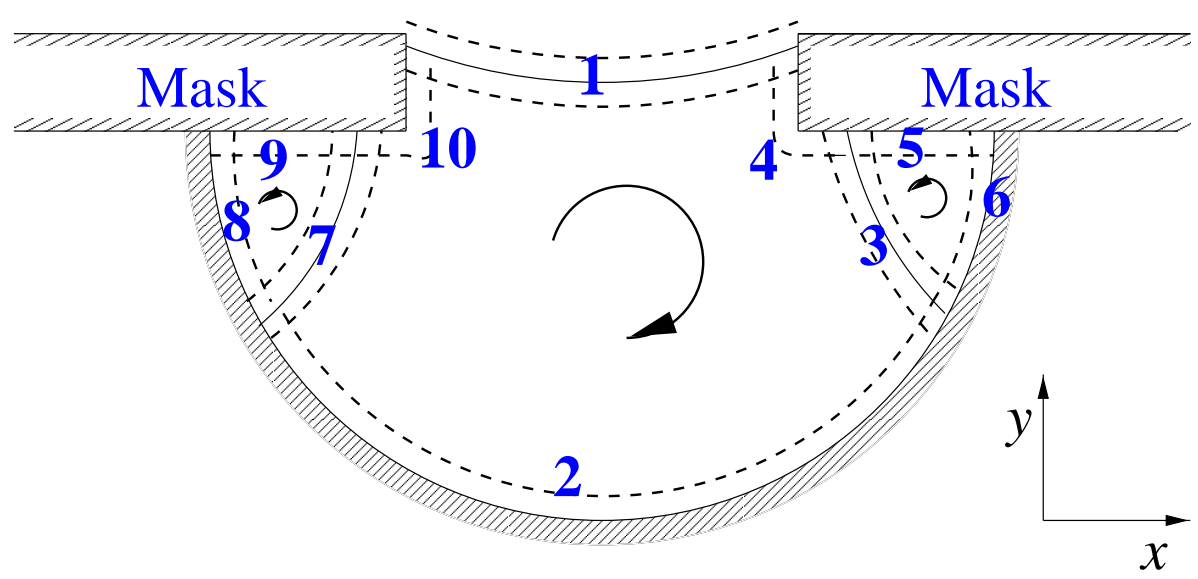


Figure 5

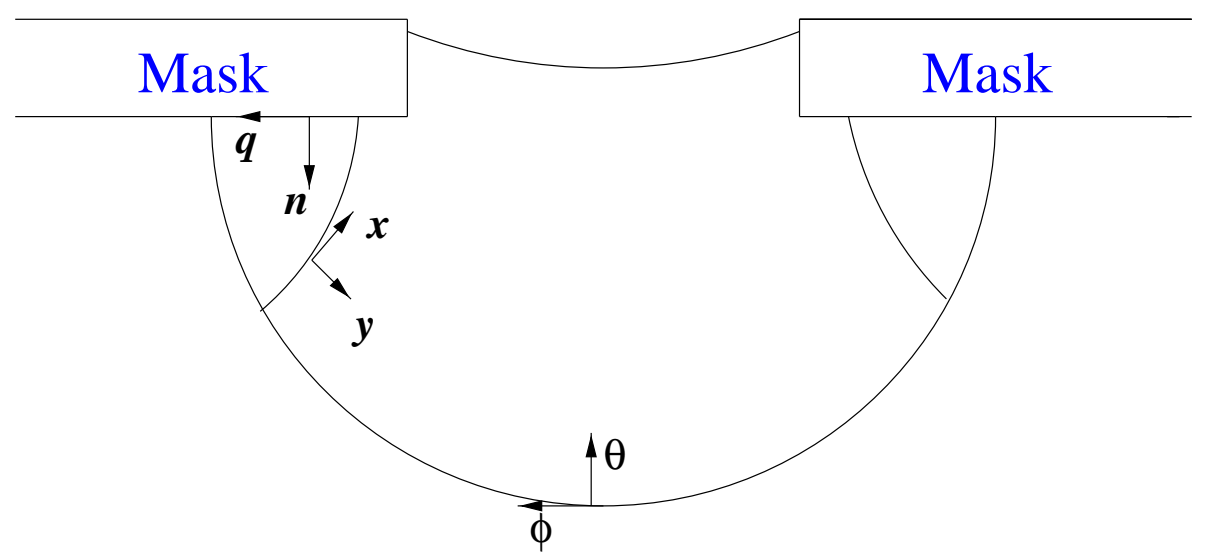


FiguRE 6

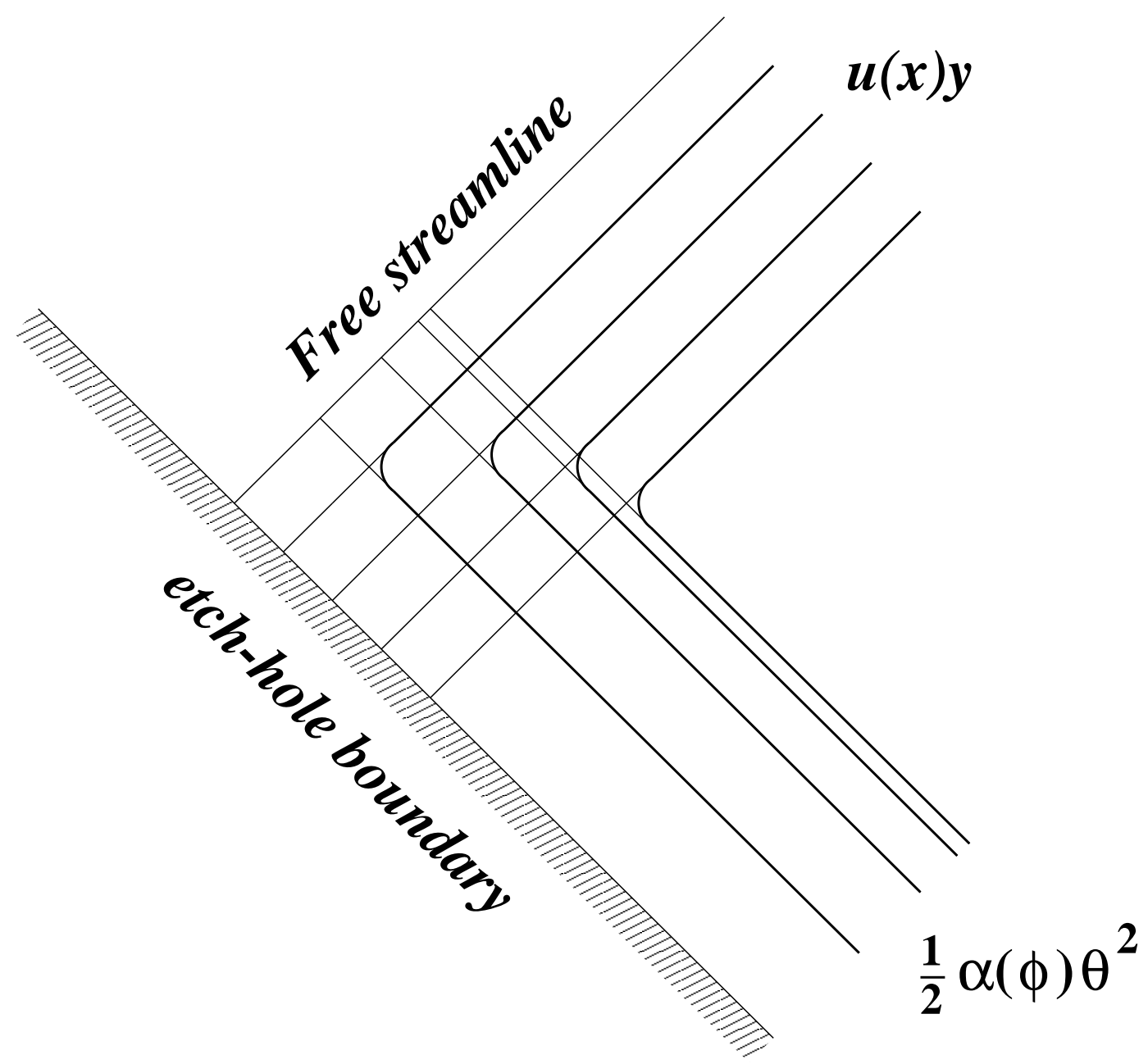


Figure 7

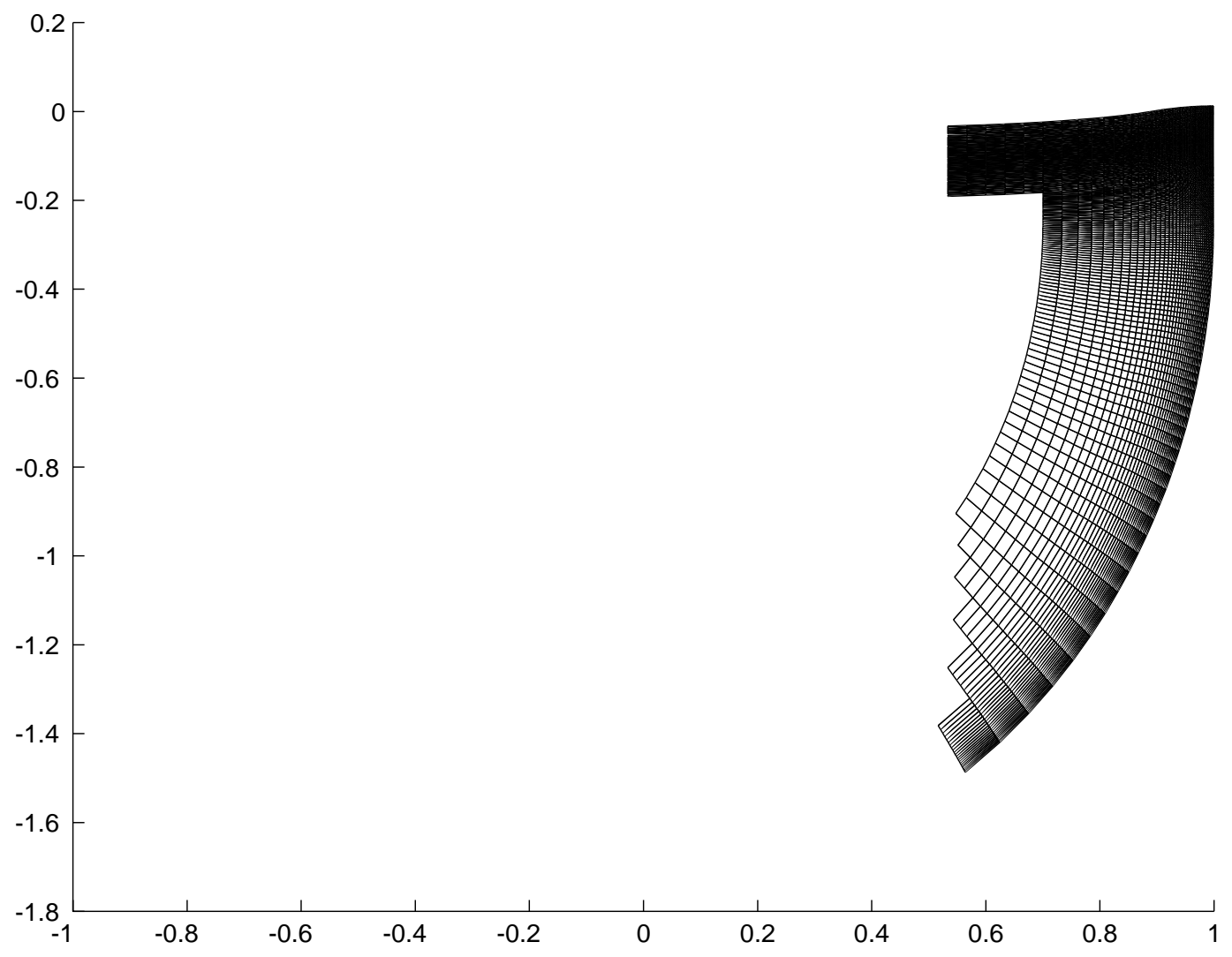

